\title{
Comprehensive analysis of IncRNA-associated competing endogenous RNA network in tongue squamous cell carcinoma
}

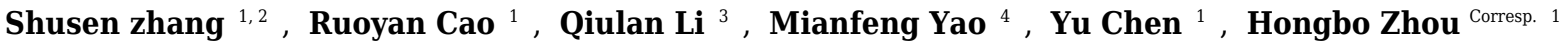 \\ ${ }^{1}$ Department of Prosthodontics, Xiangya Stomatological Hospital, Xiangya School of Stomatology, Central South University, Changsha, China \\ 2 Department of Stomatology, Hunan University of Medicine, Hunan, China \\ 3 Department of Oral Medicine, The Second Xiangya Hospital, Central South University, Changsha, China \\ 4 Department of Oral Medicine, Xiangya Hospital, Central South University, Changsha, China \\ Corresponding Author: Hongbo Zhou \\ Email address: zhb2540@csu.edu.cn
}

Background. Increasing evidence has demonstrated that long non-coding RNAs (IncRNAs) play an important role in the competitive endogenous RNA (ceRNA) networks in that they regulate protein-coding gene expression by sponging microRNAs (miRNAs). However, the understanding of the ceRNA network in tongue squamous cell carcinoma (TSCC) remains limited.

Methods. Expression profile data regarding mRNAs, miRNAs and IncRNAs as well as clinical information on 122 TSCC tissues and 15 normal controls from The Cancer Genome Atlas (TCGA) database were collected. We used the edgR package to identify differentially expressed mRNAs (DEmRNAs), IncRNAs (DEIncRNAs) and miRNAs (DEmiRNAs) between TSCC samples and normal samples. In order to explore the functions of DEmRNAs, Kyoto Encyclopedia of Genes and Genomes (KEGG) pathway analysis was performed. Subsequently, a ceRNA network was established based on the identified DEIncRNAs-DEmiRNAs and DEmiRNAs-DEmRNAs interactions. The RNAs within the ceRNA network were analyzed for their correlation with overall disease survival. Finally, IncRNAs were specifically analyzed for their correlation with clinical features in the included TSCC patient samples.

Results. A total of 1867 mRNAs, 828 IncRNAs and 81 miRNAs were identified as differentially expressed in TSCC tissues ( $\mid \log _{2}$ fold change $\geq 2$; adjusted $P$ value $<0.01$ ). The resulting ceRNA network included 16 mRNAs, 56 IncRNAs and 6 miRNAs. Six out of the 56 IncRNAs were found to be associated with the overall survival in TSCC patients $(P<0.05) ; 10$ IncRNAs were correlated with TSCC progression $(P<$ 0.05).

Conclusion. Our study deepens the understanding of ceRNA network regulatory mechanisms in TSCC. Furthermore, we identified ten IncRNAs (PART1, LINC00261, AL163952.1, C2orf48, FAM87A, LINC00052, LINC00472, STEAP3-AS1, TSPEAR-AS1 and ERVH48-1) as novel, potential prognostic biomarkers and therapeutic targets for TSCC. 
1 Comprehensive analysis of IncRNA-associated competing endogenous RNA network in

2 tongue squamous cell carcinoma

3 Shusen Zhang ${ }^{1,2 *}$, Ruoyan $\mathrm{Cao}^{1 *}$, Qiulan $\mathrm{Li}^{3}$, Mianfeng $\mathrm{Yao}^{4}, \mathrm{Yu}$ Chen ${ }^{1}$, Hongbo Zhou ${ }^{1}$

41 Department of Prosthodontics, Xiangya Stomatological Hospital, Xiangya School of

5 Stomatology, Central South University, 72 Xiangya Road, Changsha, 410000, China

62 Department of Stomatology, Hunan University of Medicine, Huaihua 418000, Hunan, China

$7 \quad{ }^{3}$ Department of Oral Medicine, The Second Xiangya Hospital, Central South University, 139

8 Middle Renmin Road, Changsha, 410011, China

$9{ }^{4}$ Department of Oral Medicine, Xiangya Hospital, Central South University, 87 Xiangya Road,

10 Changsha, 410000, China

$11 *$ These authors contributed equally to this work.

12 Corresponding Author:

13 Dr. Hongbo Zhou, PhD, DDS. Department of Prosthodontics, Xiangya Stomatological Hospital,

14 Xiangya School of Stomatology, Central South University, 72 Xiangya Road, Changsha, 410083,

15 China

16 Email: zhb2540@csu.edu.cn 


\section{Abstract}

Background. Increasing evidence has demonstrated that long non-coding RNAs (lncRNAs) play an important role in the competitive endogenous RNA (ceRNA) networks in that they regulate protein-coding gene expression by sponging microRNAs (miRNAs). However, the understanding of the ceRNA network in tongue squamous cell carcinoma (TSCC) remains limited.

Methods. Expression profile data regarding mRNAs, miRNAs and lncRNAs as well as clinical information on 122 TSCC tissues and 15 normal controls from The Cancer Genome Atlas (TCGA) database were collected. We used the edgR package to identify differentially expressed mRNAs (DEmRNAs), lncRNAs (DElncRNAs) and miRNAs (DEmiRNAs) between TSCC samples and normal samples. In order to explore the functions of DEmRNAs, Kyoto Encyclopedia of Genes and Genomes (KEGG) pathway analysis was performed. Subsequently, a ceRNA network was established based on the identified DElncRNAs-DEmiRNAs and DEmiRNAs-DEmRNAs interactions. The RNAs within the ceRNA network were analyzed for their correlation with overall disease survival. Finally, lncRNAs were specifically analyzed for their correlation with clinical features in the included TSCC patient samples.

Results. A total of 1867 mRNAs, 828 lncRNAs and 81 miRNAs were identified as differentially expressed in TSCC tissues $\left(\mid \log _{2}\right.$ fold change $\mid \geqslant 2$; adjusted $\mathrm{P}$ value $\left.<0.01\right)$. The resulting ceRNA network included 16 mRNAs, 56 lncRNAs and 6 miRNAs. Six out of the $56 \operatorname{lncRNAs}$ were found to be associated with the overall survival in TSCC patients $(P<0.05) ; 10 \operatorname{lncRNAs}$ were correlated with TSCC progression $(P<0.05)$.

Conclusion. Our study deepens the understanding of ceRNA network regulatory mechanisms in 
43 TSCC. Furthermore, we identified ten lncRNAs (PART1, LINC00261, AL163952.1, C2orf48,

44 FAM87A, LINC00052, LINC00472, STEAR-AS1, TSPEAR-AS1 and ERVH48-1) as novel,

45 potential prognostic biomarkers and therapeutic targets for TSCC.

47 Introduction

48 Tongue squamous cell carcinoma (TSCC) is the most common type of oral squamous cell

49 carcinoma (OSCC) with remarkable invasiveness, early lymph node metastasis and a poor

50 prognosis (Sano \& Myers 2007; Zhou et al. 2015). The quality of life for TSCC survivors is often

51 reduced due to speech disfunction, mastication, and deglutition. Recently, the incidence and

52 mortality of TSCC have steadily risen in the United States (Siegel et al. 2014; Siegel et al. 2015;

53 Siegel et al. 2016; Siegel et al. 2017; Siegel et al. 2018). Despite significant advancements in

54 surgical excision, radiotherapy and chemotherapy, mortality rates and recurrence rates for this

55 cancer remain high (Adeel \& Suhail 2016; Chen et al. 2018). For these reasons, the molecular

56 mechanisms of TSCC tumorigenesis urgently require further study; potential biomarkers as well

57 as therapeutic targets in this cancer should be identified in order to improve clinical outcomes.

59 Long noncoding RNAs (lncRNAs) are a subclass of noncoding RNAs longer than 200 nucleotides

60 (Ponting et al. 2009). Of late, lncRNAs have been a new focus of cancer research and were found

61 to be involved in tumorigenesis and metastasis (Chen et al. 2017; Gutschner et al. 2013; Yang et

62 al. 2017; Yang et al. 2016b; Yuan et al. 2014). In TSCC, lncRNAs were reported to act as

63 oncogenes or tumor suppressors and affect patient prognosis. For example, knockdown of AFAP1- 
64 AS1 could suppress cell proliferation, migration and invasion in TSCC (Wang et al. 2018).

Additionally, the overexpression of lncRNA MEG3 inhibited cell proliferation and induced apoptosis in TSCC (Jia et al. 2014). The current literature has demonstrated that lncRNAs regulate gene expression via genetic imprinting, splicing regulation, chromatin remodeling, mRNA decay, and translational regulation (Zhu et al. 2013). However, the formation and development of tumors is a complex pathophysiological process. The mechanisms by which lncRNAs affect TSCC biology remain unelucidated.

Salmena et al. proposed the competing endogenous RNA (ceRNA) hypothesis, which stated that lncRNA could crosstalk with mRNA by sharing common microRNA response elements (MREs) with miRNA (Salmena et al. 2011). More and more studies have validated the involvement of ceRNA crosstalk in the development and progression of various tumors, such as those of breast cancer, hepatocellular cancer and pancreatic cancer. Some of the few relevant studies on such crosstalk in TSCC have been verified, such as LINC00511/ miR-765/ LAMC2 (Ding et al. 2018) and H19/ let-7a/ HMGA2 (Kou et al. 2018). Furthermore, comprehensive analysis of TSCCassociated lncRNAs and miRNAs in a whole genome wide context is lacking, especially based on high-throughput sequencing with a large-scale sample size.

2 To better understand how lncRNAs regulate gene expression by sponging miRNAs in TSCC, we build a ceRNA network based on the TCGA database, including 6 mRNAs, 56 lncRNAs and 6 miRNAs. In addition, we found 10 lncRNAs to be associated with survival and 10 lncRNAs having 
85

86

87

88

89

90

91

92

an association with carcinogenesis. Results of these analyses are a starting point to analyze ceRNA crosstalk and gain insight into the molecular mechanisms participating in the tumorigenesis and progression of TSCC.

\section{Materials and Methods}

\section{Patients and samples}

RNA sequencing data and the corresponding clinical information for our TSCC dataset were retrieved from the TCGA data portal. The inclusion criteria were set as follows: 1) patients with follow-up survival times less than 2000 days; 2) patients with detailed clinicopathological information including age, gender, survival time, survival status, pathological stage, TNM stage. As most patients were missing data about their metastatic states, we did not analyze this information. After filtering available data with our inclusion criteria, a total of 122 TSCC patients and 15 normal controls were included in our analysis. The clinical and pathological characteristics of the TSCC patients are summarized in Table 1. This study conformed with the publication guidelines provided by TCGA (https://cancergenome.nih.gov/publications/publicationguidelines) and as our data was obtained from TCGA database, approval by an ethics committee was not required.

\section{RNA sequence data processing}

Level 3 RNASeq and miRNASeq data from TSCC samples up to June 30,2018, including 122 TSCC tissues and 15 normal controls, were downloaded from the TCGA data portal. The sequence 
106 data originated from IlluminaHiSeq_RNASeq and IlluminaHiSeq_miRNASeq sequencing 107 platforms; all the data are publicly available.

108

109

Analysis of differential expression profiles

110

The Ensembl database (http://www.ensembl.org/index.html, version 89) (Aken et al. 2016) was

111

used to identify lncRNA genes from the raw expression data. We discarded previously identified

lncRNAs that were not included in this database. Differential expression analysis of mRNAs

113 (DEmRNAs), miRNAs (DEmiRNAs) and lncRNAs (DElncRNAs) between TSCC and normal

114

115

116

117

118

119

120

121

122

123

124

125

126

tissues was carried out using the edgeR package (Robinson et al. 2010). For all p values, false

discovery rate (FDR) was applied for multiple testing correction. Absolute $\log _{2} \mathrm{FC} \geqslant 2$ and the FDR $<0.01$ were used as cut-off criteria.

\section{Functional enrichment analysis}

In order to better understand the mechanisms involved in the tumorigenesis of TSCC, we conducted Gene Ontology (GO) functional enrichment analysis using DAVID (the Database for Annotation, Visualization and Integrated Discovery) with FDR $<0.01$ as the cut-off value. KEGG analysis was performed using the ClusterProfiler package in the $\mathrm{R}$ language with a cut-off value of adjusted $\mathrm{p}$ value $<0.05$.

\section{Construction of ceRNA network}

We used the miRcode database (Jeggari et al. 2012) to predict lncRNA-miRNA interactions, which 
127 were then combined with selected miRNAs. Secondly, TargetScan (Fromm et al. 2015),

128 miRTarBase (Chou et al. 2016) and miRDB (Wong \& Wang 2015) were used to retrieve and

129 predict the targeted mRNAs of miRNA. In order to enhance the validity of this ceRNA network,

130 we only included miRNA-targeted mRNAs present in all three databases and DEmRNAs. Finally,

131 the ceRNA network was visualized using Cytoscape 3.6.1 software. A flowchart of the ceRNA

132 network is presented in Fig 1. We also performed linear regression analysis to explore the

133 correlation between ceRNA expression levels.

\section{Statistical analysis}

136 For overall survival analysis, the log-rank test was employed to compare the difference between

137 TSCC and normal samples using Kaplan Meier survival curve. The cut-off point of expression was

138 identified using survminer package ( $\mathrm{Li}$ et al. 2018b). The edgeR package was used to screen out 139 DElncRNAs associated with clinical features, by setting cut-off criteria of absolute $\log _{2} \mathrm{FC} \geqslant 1$

140 and the FDR $<0.05$. Unless specifically stated, a p value $<0.05$ was considered to represent 141 statistical significance. All statistical analyses were performed using R software (version: 3.3 .2 ).

Results

\section{DEmRNAs in TSCC}

According to the cut-off threshold of $\log _{2} \mathrm{FC} \geqslant 2$ and $\mathrm{FDR}<0.01,717$ (38.40\%) up-regulated and

1461150 (61.60\%) down-regulated genes were identified in TSCC (Supplementary Table 1). Fig. 2

147 shows the distribution of DEmRNAs between TSCC and normal controls. The expression heat 
148 149 150

151

map of DEmRNAs is shown in Supplementary Fig. 1. Red or green represents significantly upregulated and downregulated genes, respectively.

A total of 45 significantly enriched GO terms are listed in Supplementary Table 2 that correspond to DEmRNAs. For "biological processes (BP)", the top five terms were muscle filament sliding, collagen catabolic process, extracellular matrix organization, muscle contraction and skeletal system development; for the "cellular component (CC)" ontology the top five were, extracellular region, extracellular space, proteinaceous extracellular matrix, Z disc and collagen trimer; finally, the top five "molecular function (MF)" terms were, structural constituent of muscle, extracellular matrix structural constituent, calcium ion binding, heparin binding and cytokine activity (Fig. 3).

Additionally, a total of 20 significantly enriched KEGG pathways for the identified DEmRNAs are listed in Table 2, and the top 10 KEGG pathways are shown in Fig. 3D. The cytokine-cytokine receptor interaction pathway was found to harbor the largest number of DEmRNAs (Fig. 3E).

\section{DEIncRNAs in TSCC}

Based on the cut-off criteria $(\log 2 \mathrm{FC} \geqslant 2$ and FDR $<0.01)$, we identified 828 lncRNAs aberrantly expressed in TSCC compared to normal tissues, including 517 up-regulated $(62.44 \%)$ and 311 down-regulated lncRNAs (37.56\%) (Supplementary Table 3). The distribution of all the DElncRNAs are presented as a volcano plot in Fig.4 and an expression heat map of DElncRNAs is shown in Supplementary Fig. 2. 
DEmiRNAs in TSCC

171 To build our lncRNA-miRNA-mRNA ceRNA network, we also compared miRNA expression profiles in tumor tissues with normal tissues. In total, 81 DEmiRNAs were identified, including 42 up- and 39 down-regulated miRNAs (Supplementary Table 4). A volcano plot of the related DEmiRNAs is shown in Fig. 5; a corresponding expression heat map is shown in Supplementary Fig. 3.

A dysregulated ceRNA network of lncRNA-miRNA-mRNA in TSCC was established based on the above data in order to better elucidate the role of DElncRNAs. First, the 828 DElncRNAs were retrieved from the miRcode, and 102 pairs of interacting lncRNAs and miRNAs were identified using the Perl language. Subsequently, we predicted that six DEmiRNAs could interact with 56 DElncRNAs. Then we found that these six DEmiRNAs targeted $221 \mathrm{mRNAs}$ in all three databases (TargetScan, miRTarBase and miRDB). Among the 221 targeted mRNAs, only 16 mRNAs were found in the 1867 DEmRNAs (Supplementary Fig. 4). Finally, we constructed a ceRNA network relating to TSCC by incorporating 56 DElncRNAs, 6 DEmiRNAs and 16 DEmRNAs, as shown in Fig. 6. To confirm these findings, we performed smooth curve fitting between the expression levels of the DElncRNAs and DEmRNAs included in the ceRNA network. Our results indicated a positive correlation between ceRNA expression levels. For example, LINC00472 interacted with 
190 191 192 193 194 195 196 197 198 199 200 201 202 203 204 205 206 207 208 209 210

We also contract GO and KEGG analysis to reveal the functions of the 16 DEmRNAs that were involved in the ceRNA network. Only two GO terms were significantly enriched $(P<0.05)$ (Table 3).

RNAs in the ceRNA network are related to survival

LncRNAs, miRNAs and mRNAs associated with prognosis were identified using the expression profiles of 59 lncRNAs, 6 miRNAs and 16 mRNAs in the ceRNA network using Kaplan Meier Survival Curve. As a result, ten lncRNAs (PART1, LINC00261, AL163952.1, C2orf48, FAM87A, LINC00052, LINC00472, STEAR-AS1, TSPEAR-AS1 and ERVH48-1) were observed to be significantly related to overall survival rate $(\mathrm{P}<0.05)$ (Fig. 8).

\section{IncRNAs in the ceRNA network are related to clinical features}

The 64 DElncRNAs from the ceRNA network were further analyzed to identify their correlations with clinical features. TSCC patients were divided into subgroups according to pathological stage (Stage III + IV vs. stage I + II) and TNM stage (T3 + T4 vs. T1 + T2, N2 + N3 vs. N0 + N1). We found six lncRNAs with a high expression level (LINC00355, PSORS1C3, LINC00520, AC112721.1, AL139147.1, SFTA1P) and four lncRNAs with a low expression level (HCG22, LINC00492, AL035696.1, ERVH48-1) were significantly associated with the progression of TSCC (Table 4).

\section{Discussion}


211 TSCC is the most common form of oral cancer. Dysregulated genes are considered a major cause

212 of oncogenesis and the development of TSCC. Recently, the crucial role of lncRNA in gene

213 expression regulation at three levels including transcription, post-transcription and translation has

214 attracted considerable interest. Accordingly, the ceRNA hypothesis was proposed, postulating that

215 lncRNAs could act as part of post-transcriptional gene expression control. This conclusion

216 generated new insights into the biology of cancer.

218 To better understand how lncRNA-associated ceRNA crosstalk affects TSCC, we exploited a

219 large-scale TSCC data from the TCGA database and successfully established a dysregulated

220 lncRNA-associated ceRNA network. In addition, growing evidence has indicated that lncRNAs

221 have greater potential as prognostic biomarkers than protein-coding genes due to their stronger

222 correlation with tumor status (Hauptman \& Glavac 2013). Thus, we also identified ten lncRNAs

223 (PART1, LINC00261, AL163952.1, C2orf48, FAM87A, LINC00052, LINC00472, STEAR-AS1,

224 TSPEAR-AS1 and ERVH48-1) as prognostic biomarkers for TSCC. However, there is no research

225 to clearly explain the function of AL163952.1, C2orf48, FAM87A, STEAR-AS1, TSPEAR-AS1

226 and ERVH48-1.

228 PART1 is upregulated and its higher expression is associated with poor prognosis in prostate

cancer and non-small cell lung cancer (Li et al. 2017b; Sun et al. 2018). Elevated PART1 promotes

prostate cancer cell proliferation and inhibits cell apoptosis (Sun et al. 2018). On the contrary, we 
232 which was consistent with findings in OSCC (Li et al. 2017c). This may be because PART1 is

233 located on chromosome $5 q 12$, a region that is usually lost in oral squamous cell carcinoma (OSCC)

234 and head and neck squamous cell carcinoma (HNSCC) (Abou-Elhamd \& Habib 2008; Noutomi et

235 al. 2006). In our ceRNA network, low PART1 expression reduced levels of NR3C2 mediated by

236 mir-301b, and reduced expression of NR3C2 promotes tumor cell proliferation, metastasis and

237 epithelial-to-mesenchymal transition (Yang et al. 2018; Yang et al. 2016a; Zhang et al. 2017; Zhao

238 et al. 2018c). In addition, patients with low levels of NR3C2 have a poor prognosis in pancreatic

239 cancer and renal cell carcinoma (Yang et al. 2016a; Zhao et al. 2018c). The relationship between

240 mir-301b and NR3C2 has also been validated in pancreatic cancer (Yang et al. 2016a). Therefore,

241 PART1/mir-301b/NR3C2 axis may be an important mechanism that involves in TSCC

242 development.

244 LINC00261 is a tumor suppressor positively associated with prognosis in many tumors, such as

245 hepatocellular carcinoma (Zhang et al. 2018a), endometrial carcinoma (Fang et al. 2018) and non-

246 small cell lung (Liu et al. 2017). Its functions mainly include inhibiting tumor cell proliferation,

247 invasion and metastasis. LINC00261 was also down-regulated in our study. Notably, decreased

248 expression of LINC00261 indicated a better prognosis in TSCC. Our study found LINC00261

249 regulated the expression of ENPP4 and ENPP5, however, these two genes have not been

250 extensively studied. ENPP2 as their closely-related molecule has been widely reported to

251 participate in tumor development. Low expression of ENPP2 increases reactive oxygen species

252 (ROS) level, and high ROS level could promote tumor cell apoptosis (Cholia et al. 2018; Dawei 
253 et al. 2018). The special function of LINC00261 still needs to be further investigated.

254

255 Several existing studies indicate that LINC00472 plays an important role in inhibiting tumor 256

257

258

259

260

261

262

263

264

265

266

267

268

269

270

271

272

273 Considering the correlation between DElncRNAs and clinical characteristics, we found 10 
274 DElncRNAs related to pathologic stage, $\mathrm{T}$ stage and $\mathrm{N}$ stage. Though the function of these

275 IncRNAs are not well investigated, they also may be as therapeutic targets and present a new road

276 to understand the pathogenesis of TSCC. HCG22 was negatively associated with tumor stage in

277 our study. Similarly, Zhao et al. finds a significant inverse correlation between HCG22 and tumor

278 size (Zhao et al. 2018a). Regarding OSCC, HCG22 was found to be correlated with poor survival

279 basing on TCGA database. However, Feng et al. did not find a similar association using their

280 clinical data (Feng et al. 2017). LINC00355 was positively correlated with distant metastasis,

281 lymphatic metastasis and tumor stage, and negatively correlated with prognosis in colon

282 adenocarcinoma (Zhang et al. 2018b). Upregulated LINC00355 was also associated with poor

283 prognosis in prostate cancer (Jiang et al. 2018). In our study, LINC00355 was positively associated

284 with tumor stage, however, it was not identified to be a prognosis biomarker.

Another DElncRNA, SFTA1P has been reported to be a tumor suppressor by inhibiting cell

proliferation, invasion and migration in gastric cancer (Ma et al. 2018). It also increases cisplatin

chemosensitivity in lung squamous cell carcinoma; similarly, elevated SFTA1P indicates a longer

life (Li et al. 2017a). However, we found SFTA1P was up-regulated in TSCC, especially in lymph node metastasis tumor. Thus, we speculate that SFTA1P may act as an oncogene. Our ceRNA network indicated that SFTA1P up-regulated the expressions of IL-11 or HOXC8 by binding mir-

(Cai et al. 2018; Li et al. 2014) and non-small cell lung cancer (Liu et al. 2018; Zhao et al. 2018b).

Therefore, it is reasonable that our results are not consistent with other studies. Though the 
295

296

297

298

299

300

301

302

303

304

305

306

307

308

309

310

311

312

313

314

315

316

317

318

319

functions of IL-11 and HOXC8 are not validated in TSCC, our results also offer new ideas for the development of TSCC.

Our study identified some valuable lncRNAs that are associated with carcinogenesis and survival.

Few of them have been validated in vitro and in vivo, however, none of the lncRNAs were validated in TSCC. Hence, these valuable lncRNAs still need to be verified, and our ceRNA network, which was build based on high-throughput sequencing, requires further verification.

\section{Conclusion}

Taken together, we identified aberrantly expressed mRNAs, lncRNAs, and miRNAs and then successfully constructed a functional ceRNA network for TSCC tumorigenesis. Key lncRNAs should be check closely for association with survival and clinical features in TSCC patients, which provide novel lncRNAs as potential prognosis biomarkers and therapeutic targets.

\section{ACKNOWLEDGEMENTS}

We thank The Cancer Genome Atlas (TCGA) project and its contributors for this valuable public data set.

\section{REFERENCES}

Abou-Elhamd KE, and Habib TN. 2008. The role of chromosomal aberrations in premalignant and malignant lesions in head and neck squamous cell carcinoma. Eur Arch Otorhinolaryngol 265:203-207. 10.1007/s00405-0070420-z

Adeel M, and Suhail A. 2016. Squamous cell carcinoma of oral tongue in young patients - A 10 years tertiary care experience. J Pak Med Assoc 66:155-158.

Aken BL, Ayling S, Barrell D, Clarke L, Curwen V, Fairley S, Fernandez Banet J, Billis K, Garcia Giron C, Hourlier T, Howe K, Kahari A, Kokocinski F, Martin FJ, Murphy DN, Nag R, Ruffier M, Schuster M, Tang YA, Vogel JH, White S, 
Zadissa A, Flicek P, and Searle SM. 2016. The Ensembl gene annotation system. Database (Oxford) 2016. 10.1093/database/baw093

Cai WL, Huang WD, Li B, Chen TR, Li ZX, Zhao CL, Li HY, Wu YM, Yan WJ, and Xiao JR. 2018. microRNA-124 inhibits bone metastasis of breast cancer by repressing Interleukin-11. Mol Cancer 17:9. 10.1186/s12943-017-07460

Chen L, Yao H, Wang K, and Liu X. 2017. Long Non-Coding RNA MALAT1 Regulates ZEB1 Expression by Sponging miR143-3p and Promotes Hepatocellular Carcinoma Progression. J Cell Biochem 118:4836-4843. 10.1002/jcb.26158

Chen Y, Tian T, Mao MJ, Deng WY, and Li H. 2018. CRBP-1 over-expression is associated with poor prognosis in tongue squamous cell carcinoma. BMC Cancer 18:514. 10.1186/s12885-018-4249-1

Cholia RP, Dhiman M, Kumar R, and Mantha AK. 2018. Oxidative stress stimulates invasive potential in rat C6 and human U-87 MG glioblastoma cells via activation and cross-talk between PKM2, ENPP2 and APE1 enzymes. Metab Brain Dis 33:1307-1326. 10.1007/s11011-018-0233-3

Chou CH, Chang NW, Shrestha S, Hsu SD, Lin YL, Lee WH, Yang CD, Hong HC, Wei TY, Tu SJ, Tsai TR, Ho SY, Jian TY, Wu HY, Chen PR, Lin NC, Huang HT, Yang TL, Pai CY, Tai CS, Chen WL, Huang CY, Liu CC, Weng SL, Liao KW, Hsu WL, and Huang HD. 2016. miRTarBase 2016: updates to the experimentally validated miRNA-target interactions database. Nucleic Acids Res 44:D239-247. 10.1093/nar/gkv1258

Dawei H, Honggang D, and Qian W. 2018. AURKA contributes to the progression of oral squamous cell carcinoma (OSCC) through modulating epithelial-to-mesenchymal transition (EMT) and apoptosis via the regulation of ROS. Biochem Biophys Res Commun 507:83-90. 10.1016/j.bbrc.2018.10.170

Ding J, Yang C, and Yang S. 2018. LINC00511 interacts with miR-765 and modulates tongue squamous cell carcinoma progression by targeting LAMC2. J Oral Pathol Med 47:468-476. 10.1111/jop.12677

Fang Q, Sang L, and Du S. 2018. Long noncoding RNA LINC00261 regulates endometrial carcinoma progression by modulating miRNA/FOXO1 expression. Cell Biochem Funct. 10.1002/cbf.3352

Feng L, Houck JR, Lohavanichbutr P, and Chen C. 2017. Transcriptome analysis reveals differentially expressed IncRNAs between oral squamous cell carcinoma and healthy oral mucosa. Oncotarget 8:31521-31531. 10.18632/oncotarget.16358

Fromm B, Billipp T, Peck LE, Johansen M, Tarver JE, King BL, Newcomb JM, Sempere LF, Flatmark K, Hovig E, and Peterson KJ. 2015. A Uniform System for the Annotation of Vertebrate microRNA Genes and the Evolution of the Human microRNAome. Annu Rev Genet 49:213-242. 10.1146/annurev-genet-120213-092023

Gutschner T, Hammerle M, Eissmann M, Hsu J, Kim Y, Hung G, Revenko A, Arun G, Stentrup M, Gross M, Zornig M, MacLeod AR, Spector DL, and Diederichs S. 2013. The noncoding RNA MALAT1 is a critical regulator of the metastasis phenotype of lung cancer cells. Cancer Res 73:1180-1189. 10.1158/0008-5472.can-12-2850

Hauptman N, and Glavac D. 2013. Long non-coding RNA in cancer. Int J Mol Sci 14:4655-4669. 10.3390/ijms14034655

Jeggari A, Marks DS, and Larsson E. 2012. miRcode: a map of putative microRNA target sites in the long non-coding transcriptome. Bioinformatics 28:2062-2063. 10.1093/bioinformatics/bts344

Jia LF, Wei SB, Gan YH, Guo Y, Gong K, Mitchelson K, Cheng J, and Yu GY. 2014. Expression, regulation and roles of miR-26a and MEG3 in tongue squamous cell carcinoma. Int J Cancer 135:2282-2293. 10.1002/ijc.28667

Jiang T, Guo J, Hu Z, Zhao M, Gu Z, and Miao S. 2018. Identification of Potential Prostate Cancer-Related Pseudogenes Based on Competitive Endogenous RNA Network Hypothesis. Med Sci Monit 24:4213-4239. $10.12659 / \mathrm{msm} .910886$ 
361

362

363

364

365

366

367

368

369

370

371

372

373

374

375

376

377

378

379

380

381

382

383

384

385

386

387

388

389

390

391

392

393

394

395

396

397

398

399

400

401

Kou N, Liu S, Li X, Li W, Zhong W, Gui L, Chai S, Ren X, Na R, Zeng T, and Liu H. 2018. H19 facilitates tongue squamous cell carcinoma migration and invasion via sponging miR-let-7. Oncol Res. 10.3727/096504018x15202945197589

Li L, Yin JY, He FZ, Huang MS, Zhu T, Gao YF, Chen YX, Zhou DB, Chen X, Sun LQ, Zhang W, Zhou HH, and Liu ZQ. 2017a. Long noncoding RNA SFTA1P promoted apoptosis and increased cisplatin chemosensitivity via regulating the hnRNP-U-GADD45A axis in lung squamous cell carcinoma. Oncotarget 8:97476-97489. 10.18632/oncotarget.22138

Li M, Zhang W, Zhang S, Wang C, and Lin Y. 2017b. PART1 expression is associated with poor prognosis and tumor recurrence in stage I-III non-small cell lung cancer. J Cancer 8:1795-1800. 10.7150/jca.18848

Li S, Chen X, Liu X, Yu Y, Pan H, Haak R, Schmidt J, Ziebolz D, and Schmalz G. 2017c. Complex integrated analysis of IncRNAs-miRNAs-mRNAs in oral squamous cell carcinoma. Oral Oncol 73:1-9. 10.1016/j.oraloncology.2017.07.026

Li W, Lu Y, Han R, Yue Q, Song X, Wang F, Wu R, Hou F, Yang L, Xu L, Zhao R, and Hu J. 2018a. Gremlin2 Regulates the Differentiation and Function of Cardiac Progenitor Cells via the Notch Signaling Pathway. Cell Physiol Biochem 47:579-589. 10.1159/000490012

Li X, Yuan Y, Ren J, Shi Y, and Tao X. 2018b. Incremental Prognostic Value of Apparent Diffusion Coefficient Histogram Analysis in Head and Neck Squamous Cell Carcinoma. Acad Radiol 25:1433-1438. 10.1016/j.acra.2018.02.017

Li Y, Chao F, Huang B, Liu D, Kim J, and Huang S. 2014. HOXC8 promotes breast tumorigenesis by transcriptionally facilitating cadherin-11 expression. Oncotarget 5:2596-2607. 10.18632/oncotarget.1841

Liu H, Zhang M, Xu S, Zhang J, Zou J, Yang C, Zhang Y, Gong C, Kai Y, and Li Y. 2018. HOXC8 promotes proliferation and migration through transcriptional up-regulation of TGFbeta1 in non-small cell lung cancer. Oncogenesis 7:1. 10.1038/s41389-017-0016-4

Liu Y, Xiao N, and Xu SF. 2017. Decreased expression of long non-coding RNA LINC00261 is a prognostic marker for patients with non-small cell lung cancer: a preliminary study. Eur Rev Med Pharmacol Sci 21:5691-5695. 10.26355/eurrev_201712_14014

Ma H, Ma T, Chen M, Zou Z, and Zhang Z. 2018. The pseudogene-derived long non-coding RNA SFTA1P suppresses cell proliferation, migration, and invasion in gastric cancer. Biosci Rep 38. 10.1042/bsr20171193

Noutomi Y, Oga A, Uchida K, Okafuji M, Ita M, Kawauchi S, Furuya T, Ueyama Y, and Sasaki K. 2006. Comparative genomic hybridization reveals genetic progression of oral squamous cell carcinoma from dysplasia via two different tumourigenic pathways. J Pathol 210:67-74. 10.1002/path.2015

Ponting CP, Oliver PL, and Reik W. 2009. Evolution and functions of long noncoding RNAs. Cell 136:629-641. 10.1016/j.cell.2009.02.006

Robinson MD, McCarthy DJ, and Smyth GK. 2010. edgeR: a Bioconductor package for differential expression analysis of digital gene expression data. Bioinformatics 26:139-140. 10.1093/bioinformatics/btp616

Salameh A, Fan X, Choi BK, Zhang S, Zhang N, and An Z. 2017. HER3 and LINC00052 interplay promotes tumor growth in breast cancer. Oncotarget 8:6526-6539. 10.18632/oncotarget.14313

Salmena L, Poliseno L, Tay Y, Kats L, and Pandolfi PP. 2011. A ceRNA hypothesis: the Rosetta Stone of a hidden RNA language? Cell 146:353-358. 10.1016/j.cell.2011.07.014

Sano D, and Myers JN. 2007. Metastasis of squamous cell carcinoma of the oral tongue. Cancer Metastasis Rev 26:645-662. 10.1007/s10555-007-9082-y 
402

403

404

405

406

407

408

409

410

411

412

413

414

415

416

417

418

419

420

421

422

423

424

425

426

427

428

429

430

431

432

433

434

435

436

437

438

439

440

441

442

Shan Y, Ying R, Jia Z, Kong W, Wu Y, Zheng S, and Jin H. 2017. LINC00052 Promotes Gastric Cancer Cell Proliferation and Metastasis via Activating the Wnt/beta-Catenin Signaling Pathway. Oncol Res 25:1589-1599. 10.3727/096504017x14897896412027

Shen Y, Wang Z, Loo LW, Ni Y, Jia W, Fei P, Risch HA, Katsaros D, and Yu H. 2015. LINC00472 expression is regulated by promoter methylation and associated with disease-free survival in patients with grade 2 breast cancer. Breast Cancer Res Treat 154:473-482. 10.1007/s10549-015-3632-8

Siegel R, Ma J, Zou Z, and Jemal A. 2014. Cancer statistics, 2014. CA Cancer J Clin 64:9-29. 10.3322/caac.21208

Siegel RL, Miller KD, and Jemal A. 2015. Cancer statistics, 2015. CA Cancer J Clin 65:5-29. 10.3322/caac.21254

Siegel RL, Miller KD, and Jemal A. 2016. Cancer statistics, 2016. CA Cancer J Clin 66:7-30. 10.3322/caac.21332

Siegel RL, Miller KD, and Jemal A. 2017. Cancer Statistics, 2017. CA Cancer J Clin 67:7-30. 10.3322/caac.21387

Siegel RL, Miller KD, and Jemal A. 2018. Cancer statistics, 2018. CA Cancer J Clin 68:7-30. 10.3322/caac.21442

Su C, Shi K, Cheng X, Han Y, Li Y, Yu D, and Liu Z. 2018. Long Noncoding RNA LINC00472 Inhibits Proliferation and Promotes Apoptosis of Lung Adenocarcinoma Cells via Regulating miR-24-3p/ DEDD. Technol Cancer Res Treat 17:1533033818790490. 10.1177/1533033818790490

Sun M, Geng D, Li S, Chen Z, and Zhao W. 2018. LncRNA PART1 modulates toll-like receptor pathways to influence cell proliferation and apoptosis in prostate cancer cells. Biol Chem 399:387-395. 10.1515/hsz-2017-0255

Wang ZY, Hu M, Dai MH, Xiong J, Zhang S, Wu HJ, Zhang SS, and Gong ZJ. 2018. Upregulation of the long non-coding RNA AFAP1-AS1 affects the proliferation, invasion and survival of tongue squamous cell carcinoma via the Wnt/beta-catenin signaling pathway. Mol Cancer 17:3. 10.1186/s12943-017-0752-2

Wong N, and Wang X. 2015. miRDB: an online resource for microRNA target prediction and functional annotations. Nucleic Acids Res 43:D146-152. 10.1093/nar/gku1104

Wu Q, Tang SG, and Yuan ZM. 2015. Gremlin 2 inhibits adipocyte differentiation through activation of Wnt/betacatenin signaling. Mol Med Rep 12:5891-5896. 10.3892/mmr.2015.4117

Xiong D, Sheng Y, Ding S, Chen J, Tan X, Zeng T, Qin D, Zhu L, Huang A, and Tang H. 2016. LINC00052 regulates the expression of NTRK3 by miR-128 and miR-485-3p to strengthen HCC cells invasion and migration. Oncotarget 7:47593-47608. 10.18632/oncotarget.10250

Yan S, Shan X, Chen K, Liu Y, Yu G, Chen Q, Zeng T, Zhu L, Dang H, Chen F, Ling J, Huang A, and Tang H. 2018. LINC00052/miR-101-3p axis inhibits cell proliferation and metastasis by targeting SOX9 in hepatocellular carcinoma. Gene 679:138-149. 10.1016/j.gene.2018.08.038

Yang C, Ma X, Guan G, Liu H, Yang Y, Niu Q, Wu Z, Jiang Y, Bian C, Zang Y, and Zhuang L. 2018. MicroRNA-766 promotes cancer progression by targeting NR3C2 in hepatocellular carcinoma. Faseb j:fj201801151R. 10.1096/fj.201801151R

Yang S, He P, Wang J, Schetter A, Tang W, Funamizu N, Yanaga K, Uwagawa T, Satoskar AR, Gaedcke J, Bernhardt M, Ghadimi BM, Gaida MM, Bergmann F, Werner J, Ried T, Hanna N, Alexander HR, and Hussain SP. 2016a. A Novel MIF Signaling Pathway Drives the Malignant Character of Pancreatic Cancer by Targeting NR3C2. Cancer Res 76:3838-3850. 10.1158/0008-5472.can-15-2841

Yang W, Li X, Qi S, Li X, Zhou K, Qing S, Zhang Y, and Gao MQ. 2017. IncRNA H19 is involved in TGF-beta1-induced epithelial to mesenchymal transition in bovine epithelial cells through PI3K/AKT Signaling Pathway. PeerJ 5:e3950. 10.7717/peerj.3950

Yang YT, Wang YF, Lai JY, Shen SY, Wang F, Kong J, Zhang W, and Yang HY. 2016b. Long non-coding RNA UCA1 contributes to the progression of oral squamous cell carcinoma by regulating the WNT/beta-catenin 
signaling pathway. Cancer Sci 107:1581-1589. 10.1111/cas.13058

Ye Y, Yang S, Han Y, Sun J, Xv L, Wu L, Wang Y, and Ming L. 2018. Linc00472 suppresses proliferation and promotes apoptosis through elevating PDCD4 expression by sponging miR-196a in colorectal cancer. Aging (Albany NY) 10:1523-1533. 10.18632/aging.101488

Yuan JH, Yang F, Wang F, Ma JZ, Guo YJ, Tao QF, Liu F, Pan W, Wang TT, Zhou CC, Wang SB, Wang YZ, Yang Y, Yang N, Zhou WP, Yang GS, and Sun SH. 2014. A long noncoding RNA activated by TGF-beta promotes the invasion-metastasis cascade in hepatocellular carcinoma. Cancer Cell 25:666-681. 10.1016/j.ccr.2014.03.010

Zhang HF, Li W, and Han YD. 2018a. LINC00261 suppresses cell proliferation, invasion and Notch signaling pathway in hepatocellular carcinoma. Cancer Biomark 21:575-582. 10.3233/cbm-170471

Zhang Z, Che X, Yang N, Bai Z, Wu Y, Zhao L, and Pei H. 2017. miR-135b-5p Promotes migration, invasion and EMT of pancreatic cancer cells by targeting NR3C2. Biomed Pharmacother 96:1341-1348. 10.1016/j.biopha.2017.11.074

Zhang Z, Qian W, Wang S, Ji D, Wang Q, Li J, Peng W, Gu J, Hu T, Ji B, Zhang Y, Wang S, and Sun Y. 2018b. Analysis of IncRNA-Associated ceRNA Network Reveals Potential IncRNA Biomarkers in Human Colon Adenocarcinoma. Cell Physiol Biochem 49:1778-1791. 10.1159/000493623

Zhao G, Fu Y, Su Z, and Wu R. 2018a. How Long Non-Coding RNAs and MicroRNAs Mediate the Endogenous RNA Network of Head and Neck Squamous Cell Carcinoma: a Comprehensive Analysis. Cell Physiol Biochem 50:332-341. 10.1159/000494009

Zhao M, Liu Y, Liu R, Qi J, Hou Y, Chang J, and Ren L. 2018b. Upregulation of IL-11, an IL-6 Family Cytokine, Promotes Tumor Progression and Correlates with Poor Prognosis in Non-Small Cell Lung Cancer. Cell Physiol Biochem 45:2213-2224. 10.1159/000488166

Zhao Z, Zhang M, Duan X, Deng T, Qiu H, and Zeng G. 2018c. Low NR3C2 levels correlate with aggressive features and poor prognosis in non-distant metastatic clear-cell renal cell carcinoma. J Cell Physiol 233:6825-6838. 10.1002/jcp.26550

Zhou Y, Zhang L, Pan H, Wang B, Yan F, Fang X, Munnee K, and Tang Z. 2015. Bmi1 essentially mediates podocalyxinenhanced Cisplatin chemoresistance in oral tongue squamous cell carcinoma. PLoS One 10:e0123208. 10.1371/journal.pone.0123208

Zhu J, Fu H, Wu Y, and Zheng X. 2013. Function of IncRNAs and approaches to IncRNA-protein interactions. Sci China Life Sci 56:876-885. 10.1007/s11427-013-4553-6

Zhu L, Yang N, Chen J, Zeng T, Yan S, Liu Y, Yu G, Chen Q, Du G, Pan W, Li X, Zhou H, Huang A, and Tang H. 2017. LINC00052 upregulates EPB41L3 to inhibit migration and invasion of hepatocellular carcinoma by binding miR-452-5p. Oncotarget 8:63724-63737. 10.18632/oncotarget.18892 


\section{Table 1 (on next page)}

Clinicopathological characteristics of 122 patients with tongue squamous cell carcinoma 


\section{Table 1:}

2 Clinicopathological characteristics of 122 patients with tongue squamous cell carcinoma

\begin{tabular}{|c|c|c|}
\hline Characteristic & Subtype & No. of cases $(\%)$ \\
\hline \multirow[t]{2}{*}{ Age (years) } & $<60$ & $59(48.4)$ \\
\hline & $\geqslant 60$ & $63(51.6)$ \\
\hline \multirow[t]{2}{*}{ Gender } & Male & $85(69.7 \%)$ \\
\hline & Female & $37(30.3 \%)$ \\
\hline \multirow[t]{4}{*}{ Pathologic stage } & Stage I & $13(10.7 \%)$ \\
\hline & Stage II & $19(15.6 \%)$ \\
\hline & Stage III & $30(24.6 \%)$ \\
\hline & Stage IV & $60(49.1 \%)$ \\
\hline \multirow[t]{4}{*}{ Pathologic T } & $\mathrm{T} 1$ & $19(15.6 \%)$ \\
\hline & $\mathrm{T} 2$ & $42(34.4 \%)$ \\
\hline & $\mathrm{T} 3$ & $40(32.8 \%)$ \\
\hline & $\mathrm{T} 4$ & $21(17.2 \%)$ \\
\hline \multirow[t]{5}{*}{ Pathologic N } & No & $49(40.2 \%)$ \\
\hline & N1 & $17(13.9 \%)$ \\
\hline & N2 & $51(41.8 \%)$ \\
\hline & N3 & $1(0.8 \%)$ \\
\hline & $\mathrm{NX}$ & $4(3.3 \%)$ \\
\hline \multirow[t]{2}{*}{ Vital status } & Alive & $72(59 \%)$ \\
\hline & Dead & $50(41 \%)$ \\
\hline
\end{tabular}

3

4 


\section{Table 2 (on next page)}

Significantly enriched KEGG pathways regulated by DEmRNAs in tongue squamous cell carcinoma 
1 Table 2:

2 Significantly enriched KEGG pathways regulated by DEmRNAs in tongue squamous cell 3 carcinoma

\begin{tabular}{lllll}
\hline ID & Description & pvalue & p.adjust & Count \\
\hline hsa04970 & Salivary secretion & $2.93 \mathrm{E}-12$ & $8.40 \mathrm{E}-10$ & 31 \\
hsa04974 & Protein digestion and absorption & $9.63 \mathrm{E}-11$ & $1.38 \mathrm{E}-08$ & 29 \\
hsa04512 & ECM-receptor interaction & $3.42 \mathrm{E}-08$ & $3.27 \mathrm{E}-06$ & 24 \\
hsa04060 & Cytokine-cytokine receptor interaction & $2.38 \mathrm{E}-06$ & 0.000171 & 49 \\
hsa04020 & Calcium signaling pathway & $8.26 \mathrm{E}-06$ & 0.000402 & 34 \\
hsa04510 & Focal adhesion & $8.41 \mathrm{E}-06$ & 0.000402 & 36 \\
hsa00500 & Starch and sucrose metabolism & $2.25 \mathrm{E}-05$ & 0.000924 & 12 \\
hsa04261 & Adrenergic signaling in cardiomyocytes & $5.94 \mathrm{E}-05$ & 0.00213 & 27 \\
hsa05414 & Dilated cardiomyopathy (DCM) & 0.000147 & 0.004373 & 19 \\
hsa05410 & Hypertrophic cardiomyopathy (HCM) & 0.000152 & 0.004373 & 18 \\
hsa04976 & Bile secretion & 0.00022 & 0.00573 & 16 \\
hsa03320 & PPAR signaling pathway & 0.000363 & 0.008688 & 16 \\
hsa04973 & Carbohydrate digestion and absorption & 0.000833 & 0.018399 & 11 \\
hsa04971 & Gastric acid secretion & 0.001295 & 0.026541 & 15 \\
hsa04260 & Cardiac muscle contraction & 0.001957 & 0.035163 & 15 \\
hsa00910 & Nitrogen metabolism & 0.00196 & 0.035163 & 6 \\
hsa04964 & Proximal tubule bicarbonate reclamation & 0.002199 & 0.035586 & 7 \\
hsa04610 & Complement and coagulation cascades & 0.002232 & 0.035586 & 15 \\
hsa05146 & Amoebiasis & 0.002566 & 0.038758 & 17 \\
hsa00830 & Retinol metabolism & 0.003408 & 0.048901 & 13 \\
\hline
\end{tabular}




\section{Table 3 (on next page)}

GO terms enriched by 16 DEmRNAs that were involved in the ceRNA network 
1 Table 3: GO terms enriched by 16 DEmRNAs that were involved in the ceRNA network

\begin{tabular}{cccc}
\hline Category & Term & PValue & Genes \\
\hline GOTERM_BP_FAT & $\begin{array}{c}\text { GO:0007167 enzyme } \\
\text { linked receptor protein } \\
\text { signaling pathway } \\
\text { GO:0030509 BMP } \\
\text { signaling pathway }\end{array}$ & 0.0161 & CHRDL1, PTPRT, \\
GREM2 & CHRDL1, GREM2 \\
\hline
\end{tabular}

2 


\section{Table 4(on next page)}

The correlations between DEIncRNAs in the ceRNA network and clinical characteristics of tongue squamous cell carcinoma 
1 Table 4

2 The correlations between DEIncRNAs in the ceRNA network and clinical characteristics of

3 tongue squamous cell carcinoma

\begin{tabular}{lll}
\hline Comparisons & Downregulated & Upregulated \\
\hline $\begin{array}{l}\text { Pathologic Stage } \\
\text { Stage III + IV vs. stage I + II) }\end{array}$ & HCG22, LINC00492, & LINC00355, PSORS1C3 \\
$\begin{array}{ll}\text { Pathologic_T } \\
(\mathrm{T} 3+\text { T4 vs. T1 + T2) }\end{array}$ & ERVH48-1 & \\
Pathologic_N & & LINC00520, PSORS1C3 \\
$(\mathrm{N} 2+$ N3 vs. N0+ N1) & LINC00492, ERVH48-1 & AC112721.1, AL139147.1,
\end{tabular}

4

5 


\section{Figure 1}

\section{Flow chart of the ceRNA network construction.}

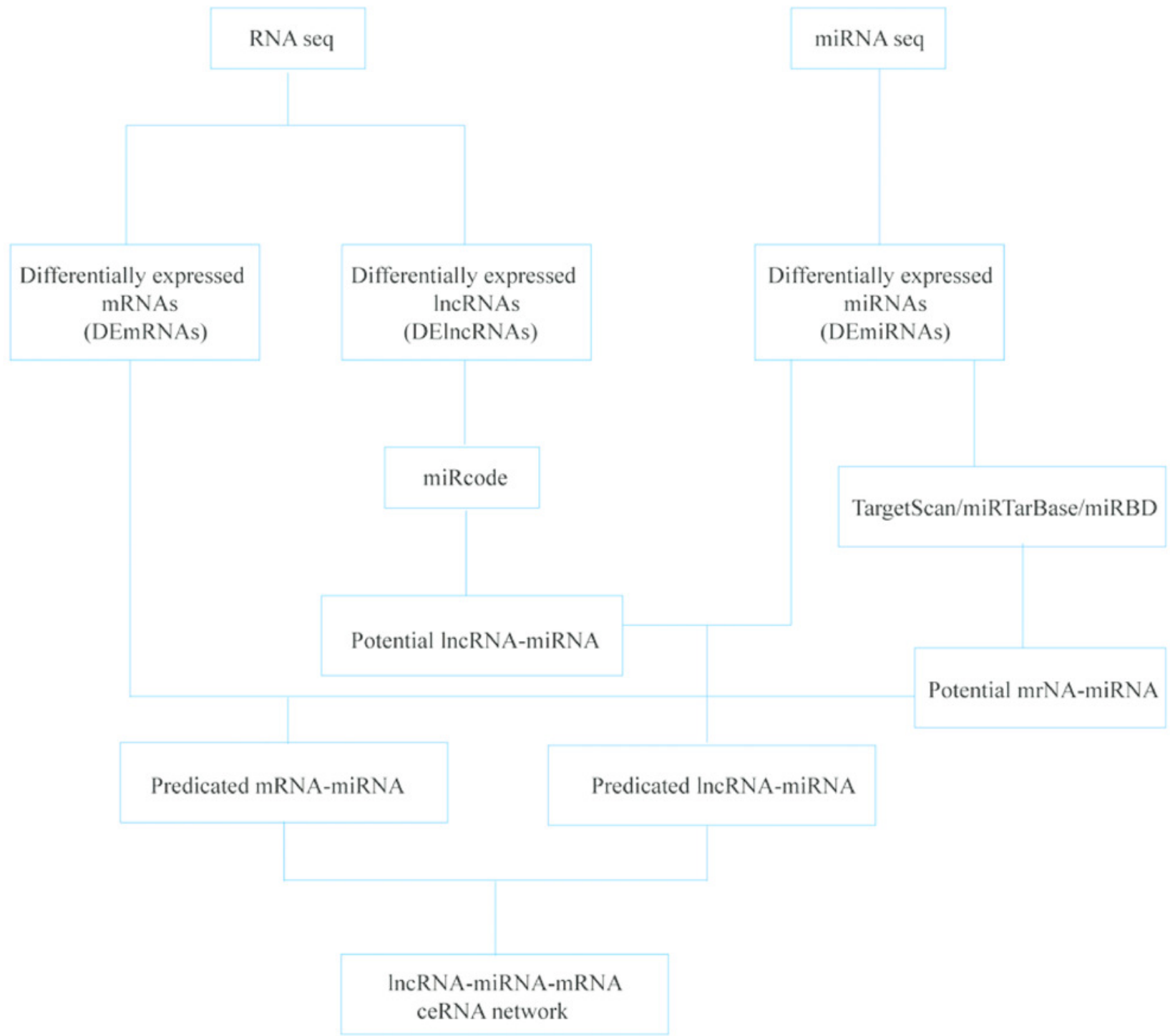


Figure 2

Volcano map of DEmRNAs.

Red spots represent up-regulated genes, and green spotsrepresent down regulated genes.

\section{Volcano}

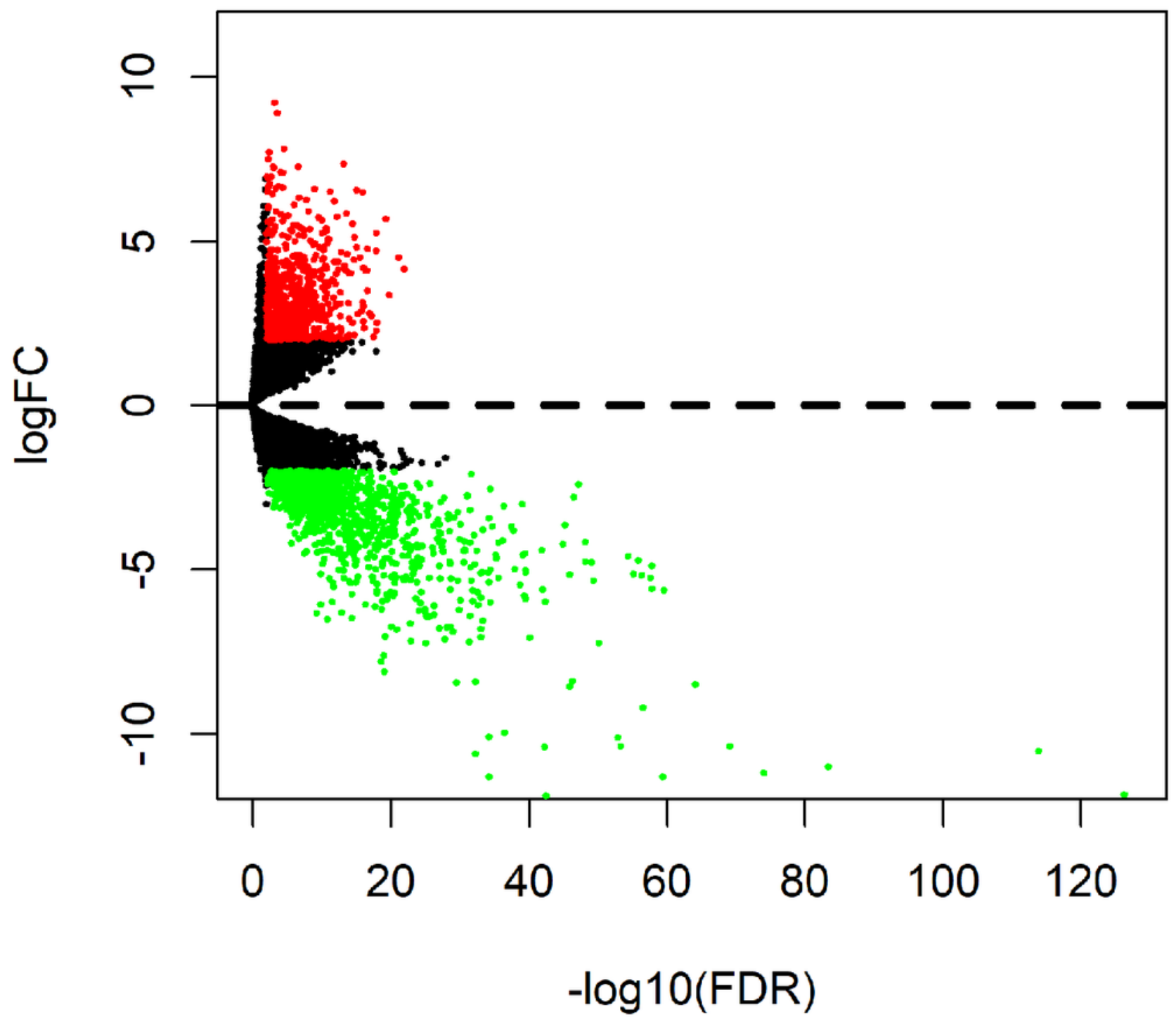




\section{Figure 3 (on next page)}

GO and KEGG pathway analyses

(A) Top 5 cellular component terms of dysregulated genes in the GO analysis. (B) Top 5 biological processes terms of dysregulated genes in the $\mathrm{GO}$ analysis. (C) Top 5 molecular function terms of dysregulated genes in the GO analysis. (D) Top10 pathways of dysregulated genes in the pathway analysis. (E) Cytokinecytokine receptor interaction map from KEGG analysis. 
A

Cellular component G0.0050018 Z disc

GO:0005615 extracellular space

GO:0005581 collagen trimer

GO:0005578 proteinaceous extracellular matrix

GO:0005576 extracellular region

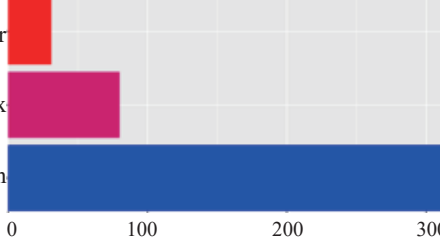
- $\log 10$ (PValue)

$\mathrm{C}$

Molecular function

GO:0008307 structural constituent of muscle

GO:0008201 heparin binding

GO:0005509 calcium ion binding

GO:0005201 extracellular matrix structural constituent

GO:0005152 cytokine activity
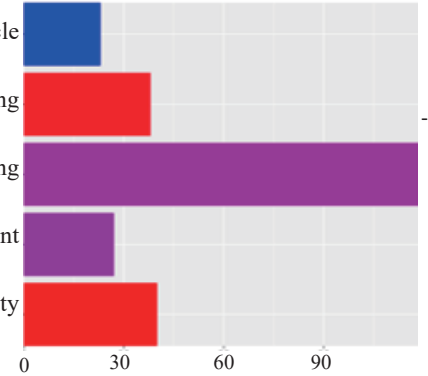

E

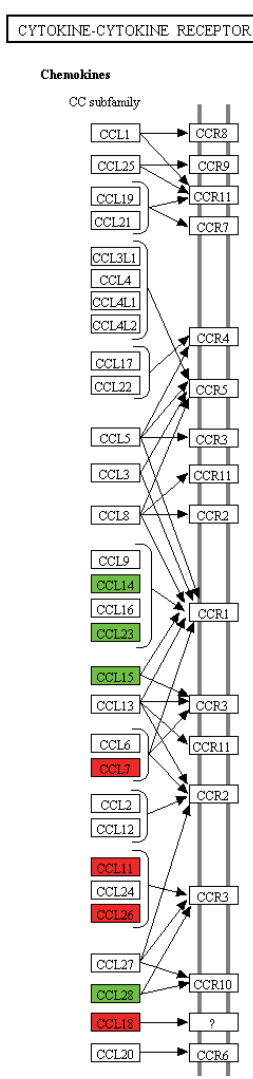

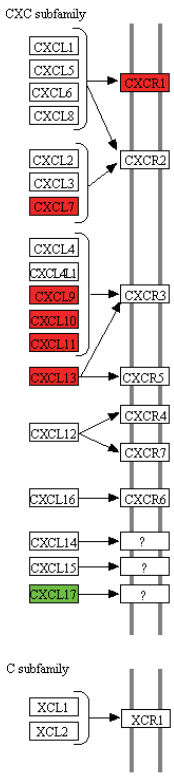

CX3C subfamily

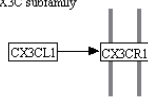

The class I helical cytolines
y-chain utilising
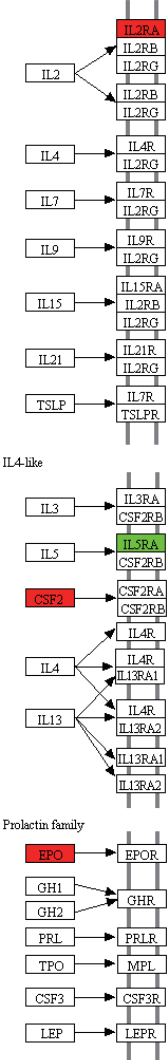

Biological processes cript to be reviewed

GO:0030574 collagen catabolic process

GO:0030198 extracellular matrix organization

GO:0030049 muscle filament slidin

GO:0006936 muscle contraction

GO:0001501 skeletal system development

Cytokine-cytokine receptor interaction Focal adhesion

Calcium signaling pathway $-\log 10$ (PValue)

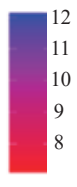

\section{Adr}

in cardiomyocytes

Protein digestion and absorption

drenergic aignaling in cardiomyocytes

ECM-receptor interaction

Dilated cardiomyopathy (DCM)

Hypertrophic cardiomyopathy (HCM)

Starch and sucrose metabolism.

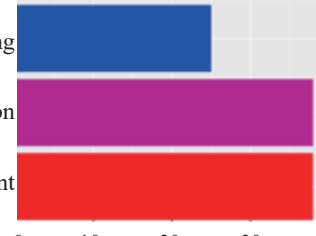

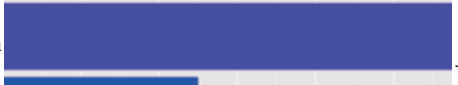

$\log 10($ PValue)
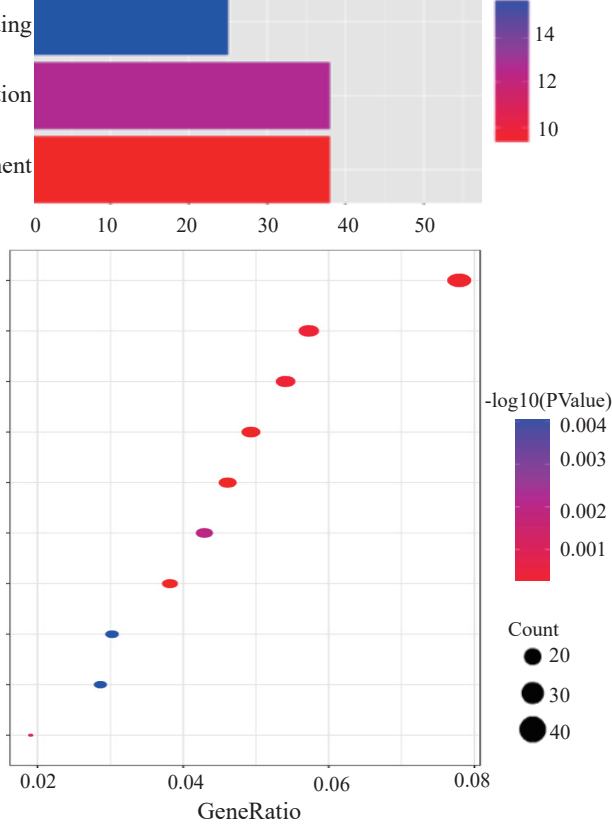

GeneRatio 
Figure 4

Volcano map of DEIncRNAs.

Red spots represent up-regulated genes, and green spotsrepresent down regulated genes.

\section{Volcano}

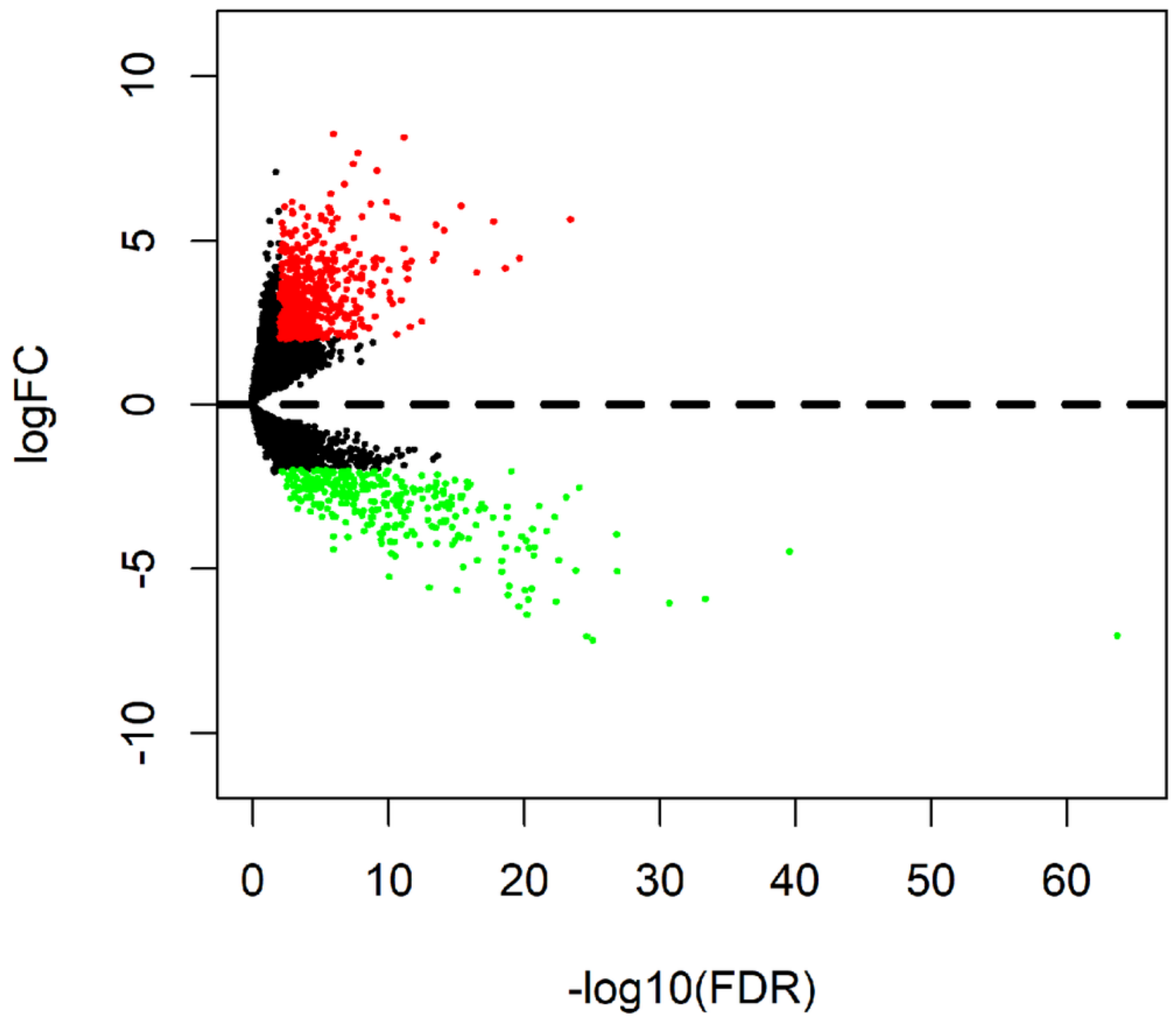


Figure 5

Volcano map of DEmiRNAs. Red spots represent up-regulated genes, and green spotsrepresent down regulated genes.

\section{Volcano}

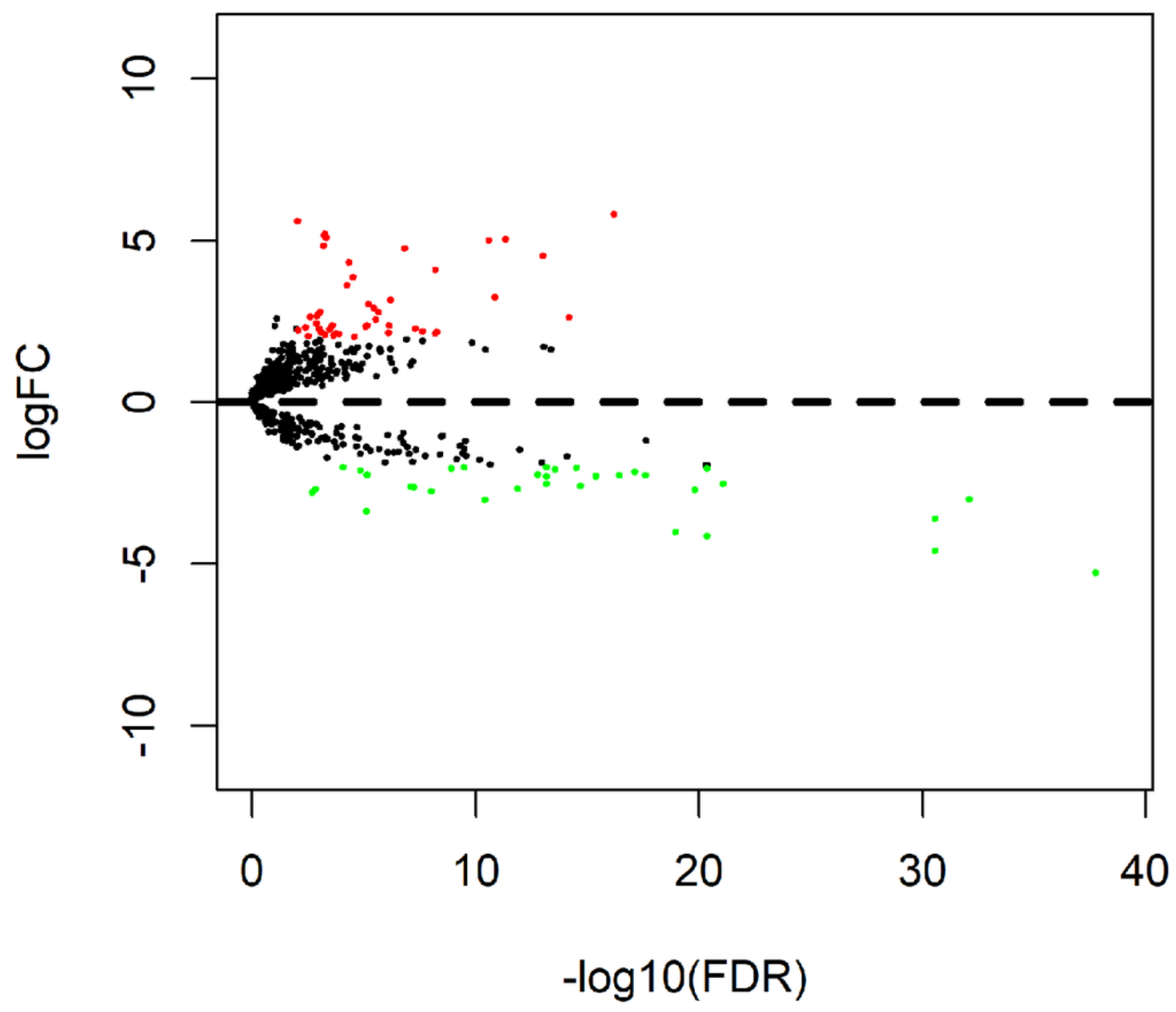


Figure 6

DEIncRNAs mediated ceRNA regulatory network in TSCC. The red nodes indicate expression up-regulation, and blue nodes indicate expression down-regulation. LncRNAs, miRNAs and mRNAs are represented by diamond, rounded rectangle, and ellipse, respectively.

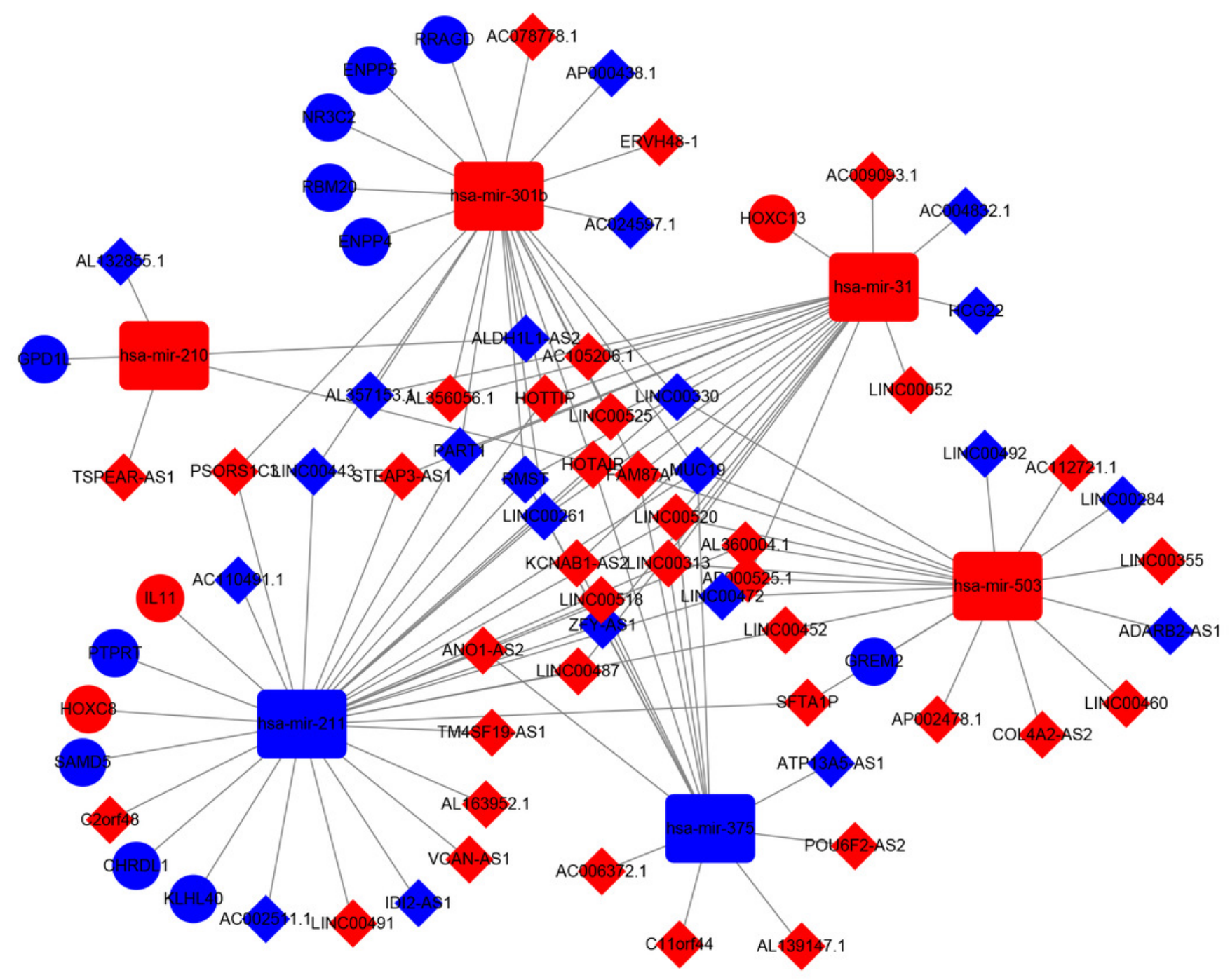


Figure 7

Linear regression of PART1 vs SAMD5 expression level.
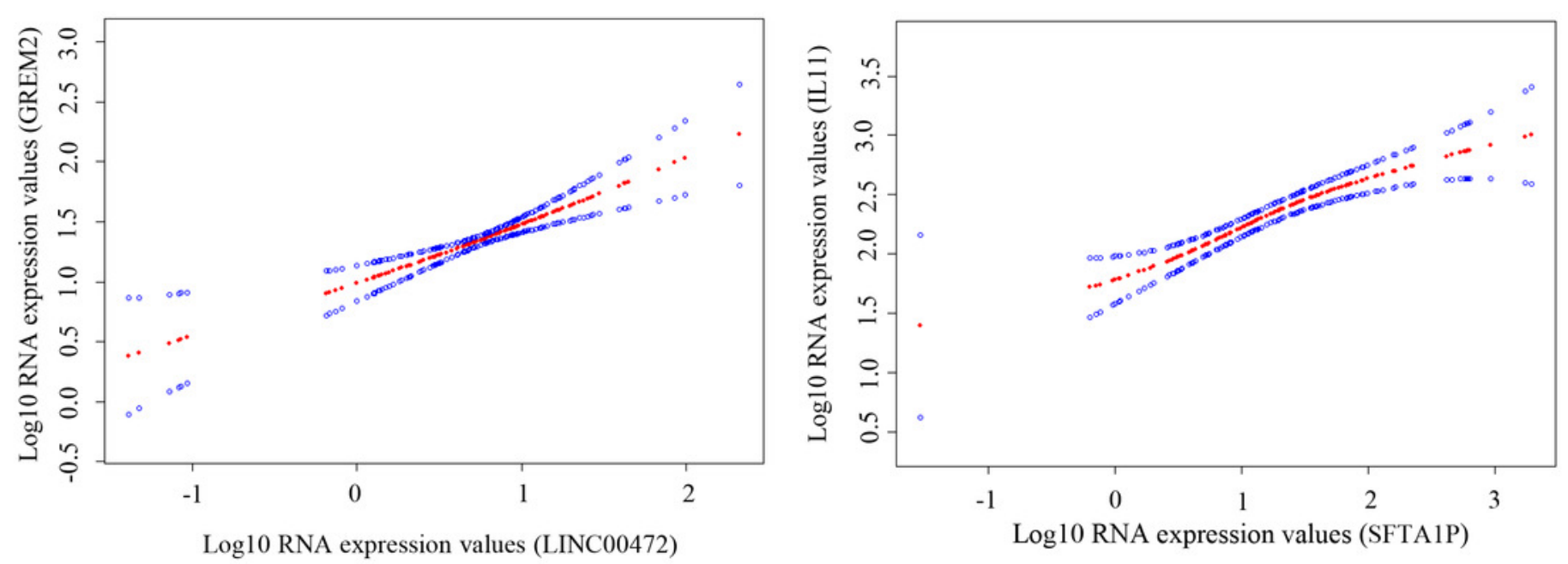
Figure 8 (on next page)

Kaplan-Meiercurve analysis of DEIncRNAs and overall survival rate in tongue squamous cellcarcinoma patients. 

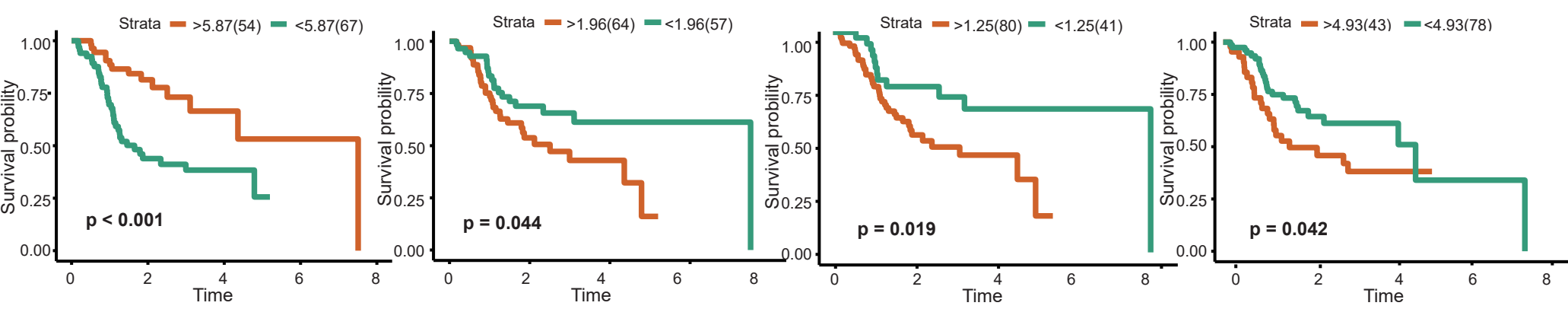

AL163952.1

C2orf48
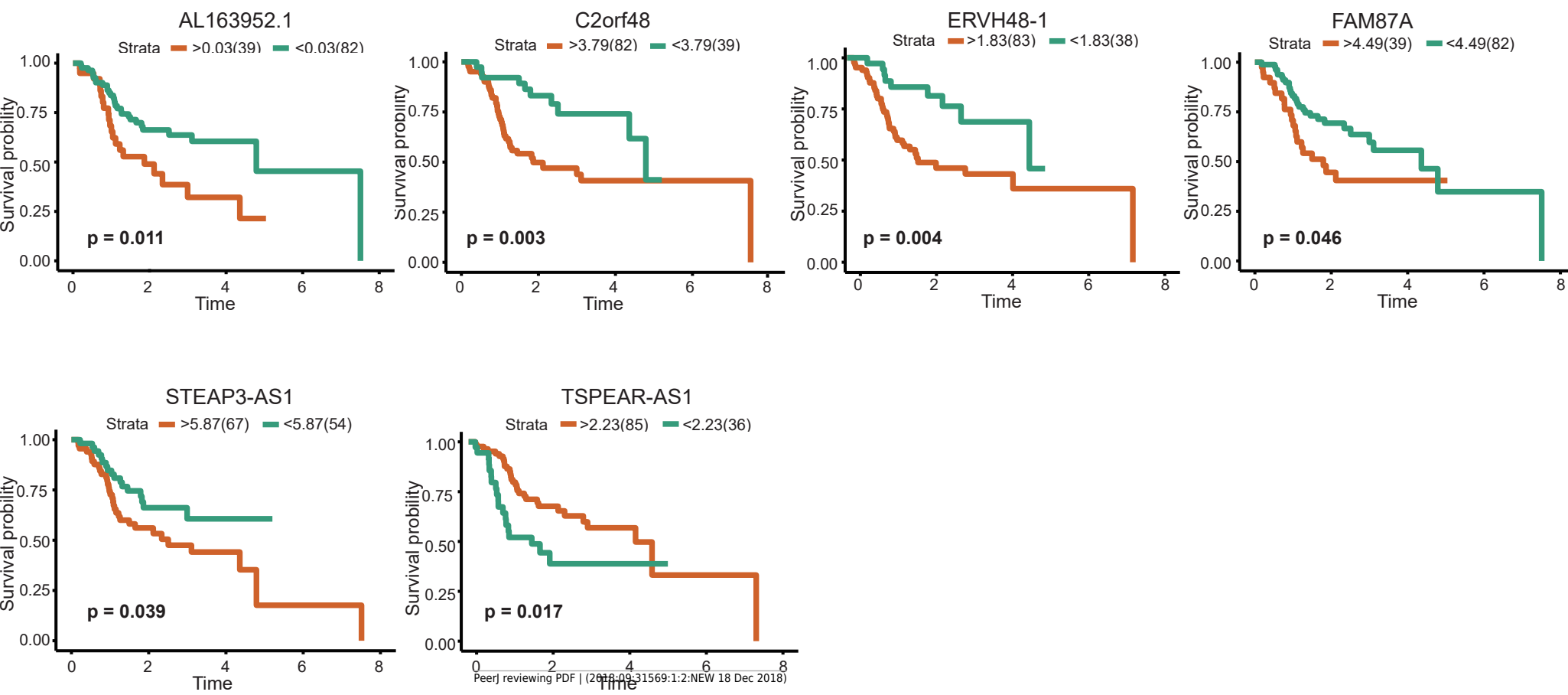